\title{
Higher dimensional black holes with a generalized gravitational action
}

\author{
Jerzy Matyjasel* and Małgorzata Telecka \\ Institute of Physics, Maria Curie-Sktodowska University \\ pl. Marii Curie-Sktodowskiej 1, 20-031 Lublin, Poland \\ Dariusz Tryniecki \\ Institute of Theoretical Physics, Wroctaw University \\ pl. M. Borna 9, 50-204 Wroctaw, Poland
}

(Dated: June 19, 2018)

\begin{abstract}
We consider the most general higher order corrections to the pure gravity action in $D$ dimensions constructed from the basis of the curvature monomial invariants of order 4 and 6 , and degree 2 and 3, respectively. Perturbatively solving the resulting sixth-order equations we analyze the influence of the corrections upon a static and spherically symmetric back hole. Treating the total mass of the system as the boundary condition we calculate location of the event horizon, modifications to its temperature and the entropy. The entropy is calculated by integrating the local geometric term constructed from the derivative of the Lagrangian with respect to the Riemann tensor over a spacelike section of the event horizon. It is demonstrated that identical result can be obtained by integration of the first law of the black hole thermodynamics with a suitable choice of the integration constant. We show that reducing coefficients to the Lovelock combination, the approximate expression describing entropy becomes exact. Finally, we briefly discuss the problem of field redefinition and analyze consequences of a different choice of the boundary conditions in which the integration constant is related to the exact location of the event horizon and thus to the horizon defined mass.
\end{abstract}

PACS numbers: 04.50.+h, 04.70.Dy

\section{INTRODUCTION}

In recent years gravitation theories with higher derivative terms have attracted a great deal of attention. Indeed, according to our present understanding the general relativity is to be treated as the lowest order term of the effective theory consisting of a series of loop or string corrections. Typically such corrections are constructed from higher powers of curvature and their derivatives,

*Electronic address: jurek@kft.umcs.lublin.pl, matyjase@tytan.umcs.lublin.pl 
and, hence, the gravitational action $I$ can be written as

$$
I=\sum_{k=0}^{m} \alpha_{k} I_{k}
$$

where $I_{k}$ for $k \geq 1$ is the contribution of operators of dimension $2 k, I_{0}$ is related to the cosmological constant and $\alpha_{k}$ are arbitrary constants. Among the higher curvature theories a great deal of activity has been focused on the Lovelock gravity [1]. In this theory, the Lagrangian $L_{m}$ is constructed from the dimensionally extended Euler densities of a $2 k$-dimensional manifold

$$
\mathcal{L}_{k}=2^{-k} \delta_{a_{1} b_{1} \ldots a_{k} b_{k}}^{c_{1} d_{1} \ldots c_{k} d_{k}} R_{c_{1} d_{1}}^{a_{1} b_{1}} \ldots R_{c_{k} d_{k}}^{a_{k} b_{k}}
$$

where the generalized $\delta$ function is totally antisymmetric in both sets of indices. A $m$-th order Lovelock action, $I_{m}$, is the sum of $m+1$ terms given by Eq. (2) of ascending complexity

$$
I_{m}=\int d^{D} x(-g)^{1 / 2} L_{m}=\int d^{D} x(-g)^{1 / 2} \sum_{k=0}^{m} \alpha_{k} \mathcal{L}_{k}
$$

where $\alpha_{k}$ are arbitrary parameters. If the Lovelock action includes the terms up to $\mathcal{L}_{m}$, the dimension of the spacetime should satisfy $D \geq 2 m+1$.

Varying the action functional $I_{m}$ with respect to the metric tensor one obtains the equations of motion of pure gravity, which can be schematically written as

$$
L^{a b}=\frac{1}{(-g)^{1 / 2}} \frac{\delta}{\delta g_{a b}} I_{m}=0 .
$$

Originally, $L_{a b}$ has been introduced by Lovelock to demonstrate the most general symmetric and divergence-free second rank tensor, which can be constructed from the metric and its first and second derivatives. Since the resulting equations of the Lovelock gravity involve at most second derivatives of the metric it avoids some of the typical problems of the higher curvature theories 22, 3]. Specifically, at the classical level, it avoids singular perturbations [4, 5, 6] which do not approach their Einsteinian counterparts as the perturbative expansion parameter is set to zero, and, when linearized, the Lovelock equations are free of ghosts. Moreover, the higher-order terms appear quite naturally as the low- energy limit of the string theory [2, 3].

At each order $\mathcal{L}_{k}$ is a linear combination of the basis curvature invariants with the particular set of coefficients calculated from Eq. (2). For example, in the first two (simplest) cases, one has a cosmological constant and the curvature scalar, for $k=0$ and $k=1$ respectively. At $k=2$ there are three invariants which are combined into the Gauss-Bonnet term whereas at $k=3$ the basis has eight members. 
On the other hand, one may consider the more general curvature terms, with arbitrary coefficients rather than those inspired by the particular theory. (See for example [7, 8] and references cited therein). In this case, the relation between dimension of the spacetime and the order of higher curvature terms retained in the action functional is lost and the dynamical equations inevitably involve higher derivatives of the metric. There is nothing wrong in using such equations, provided only the physical solutions are selected. However, the expected complexity of the resulting equations may be a serious obstacle in this regard.

The literature devoted to various aspects of the higher derivative gravity is vast indeed. As the examples of such theories one may consider the quadratic or higher order gravity (see [9, 10, 11, 12, 13, 14, 15, 16] and the references cited therein), and, when a negative cosmological constant is present, the Einstein-Hilbert action in $D=5$, supplemented with $\operatorname{Riem}^{2}=R_{a b c d} R^{a b c d}$, which corresponds to an effective $\mathrm{AdS}_{5}$ bulk action [17].

The foregoing discussion indicates that the analyses can be carried out in two directions. First, one can construct and investigate the possible candidate terms that may appear in $I_{k}$, whereas the second direction of calculations is to apply the thus constructed equations in the particular context of black hole physics or cosmology with or without additional matter fields. The latter approach has been successfully applied in various contexts in Refs. 17, 18, 19, 20, 21, 22, 23, 24, 25, 26] and in the references cited therein.

A few words of comment are in order here. First, it should be observed that we have no information on $m$, i. e., the number of terms that should be retained in Eq.(11). Second, and that is closely related to the above observation, it is really desirable and perhaps unavoidable to construct the solutions of the dynamical equations which reflect the nature of their derivation. Finally, it should be observed that because of complexity of the problem the full system of equations is probably intractable analytically and one has to construct either approximate solutions or refer to the numerical methods.

In this note we shall explore the second possibility and perturbatively solve the equations resulting from the variation of the $D$-dimensional action (11) without a cosmological constant about the Tangherlini black hole [27, 28]. In doing so the class of solutions are restricted to the admissible ones. We shall assume that the total action functional $I$ is the sum of the first three nonvanishing terms, $I_{k}$, constructed from the basis curvature invariants. That is, we assume arbitrary coefficients rather than those inspired by the particular theory. The results of this paper generalize results of Lu and Wise 20] and may be though of as a partial generalization of the analogous results obtained within the framework of the Lovelock gravity [4, 29, 30, 31, 32]. 


\section{EQUATIONS}

We shall consider the action functional being a sum of the terms (conventions are $R_{a b}=R_{a c b}^{c} \sim$ $\partial_{c} \Gamma_{a b}^{c}$, signature,,,-+++$)$

$$
\begin{gathered}
I_{1}=a \int d^{D} x(-g)^{1 / 2} R, \\
I_{2}=\int d^{D} x(-g)^{1 / 2}\left(b_{1} R^{2}+b_{2} R_{a b} R^{a b}+b_{3} R_{a b c d} R^{a b c d}\right)
\end{gathered}
$$

and

$$
\begin{aligned}
I_{3} & =\int d^{D} x(-g)^{1 / 2}\left(c_{1} R^{3}+c_{2} R R_{a}^{b} R_{b}^{a}+c_{3} R R_{a b}{ }^{c d} R_{c d}{ }^{a b}+c_{4} R_{a}^{b} R_{c}^{d} R_{b d}{ }^{a c}\right. \\
& \left.+c_{5} R_{a}^{b} R_{b}^{c} R_{c}^{a}+c_{6} R_{a}^{b} R_{b c}{ }^{d e} R_{d e}^{a c}+c_{7} R_{a b}{ }^{c d} R_{c d}{ }^{e f} R_{e f}^{a b}+c_{8} R_{c e}^{a b} R_{a f}^{c d} R_{b d}^{e f}\right),
\end{aligned}
$$

where $a=\left(16 \pi G_{D}\right)^{-1}$ and $G_{D}$ is Newton's constant. That is, we will restrict ourselves to scalar terms of order 2, 4 and 6 belonging to classes $\mathcal{R}_{2,1}^{0}, \mathcal{R}_{4,2}^{0}$ and $\mathcal{R}_{6,3}^{0}$, respectively [8]. In the course of the calculations we shall assume that the coefficients $b_{i}$ and $c_{k}$ satisfy $\left|b_{i}\right| / a<<1$ and $\left|c_{k} / b_{i}\right|<<1$ for $i=1, . ., 3$ and $k=1, \ldots, 8$, respectively. The case $b_{i} \sim c_{i}$ can be easily obtained from the former one simply by relegating the terms proportional to $b_{i} b_{j}$ from the resulting expressions.

It should be noted that depending on the dimension $D$ the curvature terms may be subjected to additional relations [8]. Moreover, for a static and spherically symmetric line element we have additional vanishing combination of the elements of the curvature basis with the coefficients depending on $D$ [7].

Although it is possible to adopt (with small modifications) the results presented in Refs. 33, 34, 35], here we proceed differently and use the Weyl method 36, 37, 38]. The line element describing the static, spherically symmetric $D=d+2$-dimensional geometry may by cast into the form

$$
d s^{2}=-f^{2}(r) d t^{2}+h^{-2}(r) d r^{2}+\frac{\delta_{i j}+x_{i} x_{j}}{1-x^{2}} r^{2} d x^{i} d x^{j} \quad i, j=2, \ldots, d+1,
$$

where $x_{i}$ are the coordinates covering maximally symmetric $d$-dimensional space. The components of Riemann tensor, the basic ingredients of our calculations, are simply

$$
\begin{gathered}
R_{i j}{ }^{k m}=r^{-2}\left(1-h^{2}\right)\left(\delta_{i}^{k} \delta_{j}^{m}-\delta_{i}^{m} \delta_{j}^{k}\right), \\
R_{i r}{ }^{j r}=-r^{-1} h h^{\prime} \delta_{i}^{j},
\end{gathered}
$$




$$
R_{t r}{ }^{t r}=-f^{-1} h\left(f^{\prime} h\right)^{\prime}
$$

and

$$
R_{t i}{ }^{t j}=-r^{-1} f_{1} h^{2} f^{\prime} \delta_{i}^{j}
$$

where the prime denotes differentiation with respect to the coordinate $r$. Upon inserting the line element into $I$ and subsequently varying the thus obtained reduced action with respect to the functions $f$ and $h$, one obtains rather complicated system of equations (not displayed here), which may be further simplified with the substitution

$$
f^{2}(r)=e^{2 \psi(r)}\left(1-\frac{2 M(r)}{r^{d-1}}\right)
$$

and

$$
h^{2}(r)=1-\frac{2 M(r)}{r^{d-1}}
$$

Except for certain combinations of the numerical coefficients $b_{i}$ and $c_{k}$ leading to the dimensionally extended Euler densities, the resulting equations of motion are still too complicated to be solved exactly. Fortunately, one can easily devise the perturbative approach to the problem, treating the higher derivative terms as small perturbations.

Now, in order to simplify calculations and to keep control of the order of terms in complicated series expansions, we shall introduce another (dimensionless) parameter $\varepsilon$, substituting $b_{i} \rightarrow \varepsilon b_{i}$ and $c_{i} \rightarrow \varepsilon^{2} c_{i}$. We shall put $\varepsilon=1$ in the final stage of calculations. For the unknown functions $M(r)$ and $\psi(r)$ we assume that they can be expanded as

$$
M(r)=\sum_{i=0}^{m} \varepsilon^{i} M_{i}(r)+O\left(\varepsilon^{m+1}\right)
$$

and

$$
\psi(r)=\sum_{i=1}^{m} \varepsilon^{i} \psi_{i}(r)+O\left(\varepsilon^{m+1}\right) .
$$

The system of differential equations $M_{i}(r)$ and $\psi_{i}(r)$ is to be supplemented with the appropriate, physically motivated boundary conditions. First, it seems natural to demand

$$
M\left(r_{+}\right)=\frac{r_{+}^{d-1}}{2},
$$


or, equivalently, $M_{0}\left(r_{+}\right)=r_{+}^{d-1} / 2$ and $M_{i}\left(r_{+}\right)=0$ for $i \geq 1$, where $r_{+}$denotes the exact location of the event horizon. Such a choice leads naturally to the horizon defined mass,

$$
\mathcal{M}_{H}=\frac{d \omega_{d}}{16 \pi G_{D}} r_{+}^{d-1}
$$

On the other hand, however, one can relate the additive integration constant with the total mass of the system as seen by a distant observer. Indeed, analysis carried out in a weak field regime indicates that the constant of integration $C$ is related to the mass $\mathcal{M}$ according to the formula

$$
C=\frac{8 \pi G_{D}}{d \omega_{d}} \mathcal{M}
$$

where

$$
\omega_{d}=\frac{2 \pi^{(d+1) / 2}}{\Gamma((d+1) / 2)}
$$

is the area of a unit d-sphere. For the function $\psi(r)$ we shall always adopt the natural condition $\psi(\infty)=0$. Since the results obtained for each set of boundary conditions are not independent and one can easily transform solution of the first type into the solution of the second type (and vice versa), we shall concentrate on the boundary conditions of the second type. A brief discussion of the consequences of the boundary conditions of the first type will be given at the end of the paper.

\section{PERTURBATIVE SOLUTION}

One expects, on general grounds, that the terms proportional to the coefficients $c_{1}, c_{2}, c_{4}$ and $c_{5}$ do not contribute to the solution. Integration of the zeroth-order equations yields, as expected, the Tangherlini solution with $M_{0}(r)=C$, whereas the first-order equations give

$$
M_{1}(r)=-\frac{2 C^{2} b_{3}}{a r^{d+1}}(d-2)(d-1)
$$

and $\psi_{1}=0$. Integration of the second-order equations, although straightforward, yields much more complicated results:

$$
\begin{aligned}
M_{2}(r) & =\frac{C^{3}(d-1)}{a r^{2+2 d}}\left[4 c_{3} d\left(5+8 d+3 d^{2}\right)-2 c_{6}\left(3-5 d^{2}-2 d^{3}\right)-2 c_{7}\left(10+16 d-21 d^{2}-7 d^{3}\right)\right. \\
& +\frac{1}{2} c_{8}\left(2+25 d-24 d^{2}-7 d^{3}\right)-\frac{8 b_{1} b_{3}}{a}(d-2)(d+1)(3 d+5)-\frac{16 b_{2} b_{3}}{a}(d-2)(d+1)(d+2) \\
& \left.-\frac{8 b_{3}^{2}}{a}(d-2)\left(4 d^{2}+19 d+9\right)\right]-\frac{C^{2}\left(d^{2}-1\right)(d+1)}{a r^{d+3}}\left[8 c_{3} d-c_{6}(2-3 d)+12 c_{7}(d-1)\right. \\
& \left.-3 c_{8}(d-1)-\frac{16 b_{1} b_{3}}{a}(d-2)-\frac{12 b_{2} b_{3}}{a}(d-2)-\frac{32 b_{3}^{2}}{a}(d-2)\right]
\end{aligned}
$$


and

$$
\begin{aligned}
\psi_{2}(r) & =-\frac{C^{2}\left(d^{2}-1\right)}{a r^{2 d+2}}\left[4 c_{3} d(3+2 d)-2 c_{6}\left(1-2 d-d^{2}\right)-6 c_{7}\left(2-2 d-d^{2}\right)\right. \\
& \left.+\frac{3}{2} c_{8}\left(2-3 d-d^{2}\right)-\frac{8 b_{1} b_{3}}{a}(d-2)(2 d+3)-\frac{8 b_{2} b_{3}}{a}(d-2)(d+2)-\frac{8 b_{3}^{2}}{a}(d-2)(2 d+5)\right] .
\end{aligned}
$$

If $b_{i} \sim c_{k}$ then the terms involving $b_{i}$ in $M_{2}(r)$ and $\psi_{2}(r)$ should be omitted. Note that for $D=4$ $(d=2)$ the function $M_{1}(r)$ is identically zero, whereas in $M_{2}(r)$ and $\psi_{2}(r)$ all the terms proportional to $b_{i} b_{j}(i, j=1,2,3)$ are absent. It is because the Kretschmann scalar, $R_{a b c d} R^{a b c d}$, can be relegated from the action as the functional derivative of the Gauss-Bonnet term with respect to the metric is zero.

The line element (8) with (21,23) provides the most general solution to the problem. Since the partial results referring to a particular dimension and/or the concrete form of the action exist in the literature, it is worthwhile to compare them with our solution. For $D=4$ our solution reduces to that which can be easily constructed by integrating equations derived by Lu and Wise 20]. Similarly, retaining in the action functional (7) only the term proportional to $c_{7}$ and making substitutions $C=M / M_{P}^{2}, a=M_{P}^{2} / 16 \pi$ and $c_{7}=\alpha / M_{P}^{2}$, where $M_{P}$ is the Planck mass and $\alpha$ is a coupling constant, one obtains results presented in Ref. [21], however, with one reservation: We have not observed any difference between the values of the radii at which $g_{00}$ and $g^{11}$ vanish, that is, of course, in concord with the general form of the adopted line element.

Before proceeding further let us compare our solutions to the analogous solutions of the Lovelock gravity. First, let us introduce $a=\alpha_{1}, b_{i}=\alpha_{2} \tilde{b}_{i}$ and $c_{i}=\alpha_{3} \tilde{c}_{i}$, where $\alpha_{2}$ and $\alpha_{3}$ are the numerical coefficients that stand in front of the Gauss-Bonnet and the third-order term of the Lovelock Lagrangian, whereas $\tilde{b}_{i}$ and $\tilde{c}_{k}$ are the coefficients that give rise to the Lovelock terms. Numerically one has $\tilde{b}_{1}=\tilde{b}_{3}=-\tilde{b}_{2} / 4=1$ and $\tilde{c}_{1}=1, \tilde{c}_{2}=-12, \tilde{c}_{3}=3, \tilde{c}_{4}=24, \tilde{c}_{5}=16, \tilde{c}_{6}=-24, \tilde{c}_{7}=2, \tilde{c}_{8}=$ -8. Inserting the above coefficients into the solution, after massive simplifications, one obtains $h^{2}(r)=f^{2}(r)$ (or, equivalently $\psi(r)=0$ ) and

$$
\begin{aligned}
h^{2}(r) & =1-\frac{16 \pi G_{D} \mathcal{M}}{d \omega_{d} r^{d-1}}+4096 \frac{\pi^{3} G_{D}^{3} \mathcal{M}^{2}}{d^{2} \omega_{d}^{2} r^{2 d}} \alpha_{2}\left[1-512 \frac{\pi^{2} G_{D}^{2} \mathcal{M}}{d \omega_{d} r^{d+1}}(d-2)(d-1)\right](d-2)(d-1) \\
& +\alpha_{3} 65536 \frac{\pi^{4} G_{D}^{4} \mathcal{M}^{3}}{d^{3} \omega_{d}^{3} r^{3 d+1}}(d-4)(d-3)(d-2)(d-1) .
\end{aligned}
$$

On the other hand, although the action of the Lovelock gravity looks rather complicated it is possible to construct an exact solution describing static and spherically symmetric configuration 29 , 30, 31]. Such a solution can be found, after the substitution $f^{2}=h^{2}=1-r^{2} F(r)$, by solving for 
real roots of the $m-t h$ order polynomial equation

$$
\sum_{k=0}^{m} \hat{c}_{k} F^{k}=\frac{2 C}{r^{d+1}}
$$

where the coefficients $\hat{c}_{i}$ are given by

$$
\hat{c}_{0}=\frac{\alpha_{0}}{a} \frac{1}{d(d+1)}, \quad \hat{c}_{1}=1
$$

and

$$
\hat{c}_{k}=\frac{\alpha_{k}}{a} \prod_{n=3}^{2 k}(d+2-n) \text { for } k>1 .
$$

Note that if the cosmological constant is taken to be zero then $\alpha_{0}=0$. Finally, assuming $\alpha_{2}=b \sim \varepsilon$ and $\alpha_{3}=c \sim \varepsilon^{2}$ and expanding Eq. (25) in powers of $\varepsilon$, one easily reproduces Eq. (24).

\section{TEMPERATURE AND ENTROPY}

The approximate location of the event horizon of the black hole solution derived in the previous section is given by

$$
\begin{aligned}
r_{+} & =(2 C)^{1 /(d-1)}-\frac{d-2}{a} b_{3}(2 C)^{-1 /(d-1)}-\frac{1}{a}(2 C)^{-3 /(d-1)}\left\{c_{3}\left(d^{2}-1\right) d+\frac{1}{2} c_{6}\left(d^{2}-1\right)(d-1)\right. \\
& -\frac{1}{2} c_{7}[2-(d-1)(5 d-4) d]+\frac{1}{8} c_{8}\left(10-13 d+12 d^{2}-5 d^{3}\right)-\frac{2}{a}\left(b_{1} b_{3}+b_{2} b_{3}\right)\left(d^{2}-1\right)(d-2) \\
& \left.+\frac{1}{2 a} b_{3}^{2}(d-2)(4+6 d-13 d)\right\} .
\end{aligned}
$$

For $D=4$, the first order terms are absent and one easily reproduces Lu-Wise result

$$
r_{+}=2 G M\left[1-\frac{\pi}{G^{3} M^{4}}\left(6 c_{3}+5 c_{7}+\frac{3}{2} c_{6}-c_{8}\right)\right] .
$$

As is well-known, the Hawking temperature, $T$, can be easily calculated from the metric tensor without referring to the field equations. The standard method of obtaining $T$ relies on the Wick rotation. The Euclidean line element has no conical singularity provided the time coordinate is periodic with a period $P$ given by

$$
P=4 \pi \lim _{r \rightarrow r_{+}}\left(g_{t t} g_{r r}\right)^{1 / 2}\left(\frac{d}{d r} g_{t t}\right)^{-1} .
$$

Its reciprocal is identified with the black hole temperature, which, in the case on hand, reads

$$
\begin{aligned}
T & =\frac{d-1}{4 \pi}(2 C)^{-1 /(d-1)}-\frac{(d-2)(d-1) d}{4 \pi a} b_{3}(2 C)^{-3 /(d-1)} \\
& +\frac{d-1}{4 \pi a}(2 C)^{-5 /(d-1)}\left\{-\frac{c_{7}}{2}(d-4)[2+(d-2)(d-1) d]\right. \\
& \left.+\frac{c_{8}}{8}(d-4)[4+(d-5)(d-1) d]-\frac{b_{3}^{2}}{2 a}(d-2)^{2}\left(4+7 d-4 d^{2}\right)\right\} .
\end{aligned}
$$


It should be noted that the Hawking temperature does not depend on $b_{1}, b_{2}, c_{3}$ and $c_{6}$. This behavior can be traced back to the possibility of removing curvature terms proportional to this very coefficients by means of the appropriate field redefinition. Such a redefinition certainly modifies equations of motion of the test particles but should not modify the temperature, which, in turn, is to be measured at infinity. On the other hand, the horizon defined mass leads to the expression for temperature which depends on the full set of parameters. The reason is that the horizon defined mass is not the mass measured at infinity. We shall return to this problem later.

From Eq. (31) one sees that the heat capacity $C_{B H}=\partial \mathcal{M} / \partial T$ calculated for the Tangherlini black hole is given by

$$
C_{B H}^{T}=-\frac{d \omega_{d}}{4 G_{D}}\left(\frac{d-1}{4 \pi T}\right)^{d}
$$

and is always negative. That means that for $d>1$ such a black hole is thermodynamically unstable, i.e., it increases its temperature when radiating. On the other hand, for $T>>1$ the higher derivative corrections can modify this behaviour, and, depending on the signs and values of the coupling constants they can give a nonnegative contribution to the total heat capacity as can be easily inferred from

$$
C_{B H}=C_{B H}^{T}+\Delta C_{B H}
$$

where

$$
\begin{aligned}
\Delta C_{B H}= & \frac{\omega_{d}}{4 a G_{D}}(d-3)(d-2) d^{2}\left(\frac{d-1}{4 \pi T}\right)^{d-2} b_{3}+\frac{d \omega_{d}}{8 a G_{D}}(d-5)(d-4)\left(\frac{d-1}{4 \pi T}\right)^{d-4} \\
& \times\left\{\frac{1}{a}(d-2)^{2}(d+1)^{2} b_{3}^{2}+\frac{1}{4}[8+4(d-a)(d-1) d] c_{7}-\frac{1}{4}[4-(d-5)(d-1) d] c_{8}\right\} .
\end{aligned}
$$

It should be noted, however, that the validity of Eq. (34) has its limitations. Indeed, for small black holes the effect of the back reaction should be taken into account and the perturbative approach fails to be accurate. Similarly, since the black hole is treated as a system to which a thermal description applies, one must require that the change of the Hawking temperature caused by the emission of a single quantum of radiation is small. As discussed in Ref 39, 40] the condition for the thermal description to be self-consistent is

$$
\left|\frac{\partial T}{\partial \mathcal{M}}\right|<<1
$$


Now, making use of (31) one has

$$
\begin{aligned}
\frac{\partial T}{\partial \mathcal{M}} & =\frac{4 G_{D}}{d \omega_{d}}(2 C)^{-d / d-1}+\frac{12 G_{D} b_{3}}{a d \omega_{d}}(2 C)^{-(d+2) /(d-1)} d(d-2) \\
& -\frac{10 b_{3}^{2}}{a^{2} d \omega_{d}}(d-2)^{2}\left(4 d^{2}-7 d-4\right)(2 C)^{-(d+4) /(d-1)} \\
& +\frac{5}{2 a d \omega_{d}}(d-4)\left[4\left(d^{3}-3 d^{2}+2 d+2\right) c_{7}-\left(d^{3}-6 d^{2}+5 d-4\right) d_{8}\right](2 C)^{-(d+4) /(d-1)},
\end{aligned}
$$

and, therefore, one concludes that mini black holes violate the condition (35). On the other hand, for sufficiently massive black hole the first term in the right hand side of the above equation is dominant, and, consequently, the thermal description as well as the perturbative approach is legitimate. Such black holes are, however, thermodynamically unstable and their qualitative behaviour is similar to Schwarzschild black hole. Inclusion of the cosmological constant, angular momentum or electric charge changes the situation dramatically.

The entropy of the black hole may be calculated using various method. Two techniques, however, are especially well suited for calculations of the entropy in the higher derivative theories. One of them is the Wald's Noether charge approach [41, 42] whereas the second one is based on the field redefiniton [43, 44]. Here we shall follow the approach, in which $S$ is given in terms of the surface integral over the event horizon [42, 43, 45]:

$$
S=\frac{1}{4 G_{D}} A_{H}+4 \pi \int_{H} \frac{\partial \mathcal{L}}{\partial R_{a b c d}} \tilde{g}_{a c} \tilde{g}_{b d} \sqrt{(d)} g d^{d} x,
$$

where $\tilde{g}_{a b}$ denotes the metric in the subspace orthogonal to the event horizon, and, in the case on hand, $\mathcal{L}$ is the sum of the Lagrangians given by Eqs. (6) and (7). It should be noted that to calculate the entropy to the required order it suffices to retain in the line element the terms which are linear in $\varepsilon$.

The calculation of $S$ consists of three steps. First, it is necessary to express the line element in terms of $r_{+}$, which can be easily achieved by inverting relation (28). Simple calculations yield

$$
\begin{aligned}
C & =\frac{1}{2} r_{+}^{d-1}+\frac{b_{3}}{2 a}(d-2)(d-1) r_{+}^{d-3}-\frac{1}{a^{2}}(d-2)(d-1)\left(d^{2}-1\right)\left(b_{1} b_{3}+b_{2} b_{3}+3 b_{3}^{2}\right) r_{+}^{d-5} \\
& +\frac{1}{2 a}(d-1)^{2}(d+1)\left[c_{3} d+\frac{c_{6}}{2}(d-1)\right] r_{+}^{d-5}-\frac{1}{4 a}(d-1) c_{7}[2+d(d-1)(4-5 d)] r_{+}^{d-5} \\
& +\frac{1}{16 a}(d-1) c_{8}\left[10-d\left(13-12 d+5 d^{2}\right)\right] r_{+}^{d-5} .
\end{aligned}
$$

Equally well one may calculate $M(r)$ using the boundary conditions of the first kind (17) and take the limit as $r \rightarrow \infty$. Further, one has to calculate

$$
J^{a b c d}=\frac{\partial \mathcal{L}}{\partial R_{a b c d}}
$$


remembering that $J^{a b c d}$ shares all symmetries of the Riemann tensor. Finally, performing simple integration (which actually reduces to the multiplication of the result by the factor $\omega_{d} r_{+}^{d}$ ), after some algebra, one gets the desired result:

$$
\begin{aligned}
S & =\frac{A_{H}}{4 G_{d}}+4 \pi \omega_{d} r_{+}^{d} \varepsilon\left(-2 b_{1} R+2 b_{2} \delta_{p}{ }^{q} R_{q}{ }^{p}+4 b_{3} R_{t r}{ }^{t r}\right)_{\mid H}+4 \pi \omega_{d} r_{+}^{d} \varepsilon^{2}\left\{c_{3} \mathrm{Riem}^{2}\right. \\
& \left.+c_{6}\left[2\left(R_{t r}{ }^{t r}\right)^{2}+R_{p i}{ }^{q j} R_{q j}{ }^{p i}\right]+12 c_{7}\left(R_{t r}{ }^{t r}\right)^{2}+3 c_{8}\left[R_{t i}{ }^{t j} R_{r j}{ }^{r i}-\left(R_{t r}{ }^{t r}\right)^{2}\right]\right\}_{\mid H}+(\ldots),
\end{aligned}
$$

where ellipsis denote the terms which are omitted as they will not contribute to the result. All quantities are to be calculated at the event horizon and summation is assumed over repeated indices: $p, q=0,1(t, r)$ and $i, j=2 \ldots d+1$.

Now we are ready to calculate the entropy of the black hole described by Eqs. (1314) with (21)231). Substituting formulas collected in Appendix into Eq. (40)one has

$$
\begin{aligned}
S & =\frac{r_{+}^{d} \omega_{d}}{4 G_{D}}+\frac{1}{2 a G_{D}} b_{3}(d-1) d r_{+}^{d-2} \omega_{d}-\frac{\omega_{d} r_{+}^{d-4}}{2 a^{2} G_{D}}(d-2)(d-1) d(d+1)\left(b_{1} b_{3}+b_{2} b_{3}+3 b_{3}^{2}\right) \\
& +\frac{d(d-1)}{4 a G_{D}} \omega_{d} r_{+}^{d-4}\left[d(d+1) c_{3}+\frac{1}{2}\left(d^{2}-1\right) c_{6}+3 d(d-1) c_{7}-\frac{3}{4}(d-1)^{2} c_{8}\right] .
\end{aligned}
$$

As expected, the higher order corrections to the action modify the standard relation between $S$ and the area of the event horizon which is valid only for the Einstein gravity.

The entropy can also be calculated employing the first law of thermodynamics

$$
M=T d S+\sum_{i} \mu_{i} d Q_{i}
$$

where $\mu_{i}$ are the chemical potentials corresponding to the conserved charges $Q_{i}$. Making use of Eq. (42) one has

$$
\begin{aligned}
S & =\int T^{-1} d \mathcal{M}+S_{0} \\
& =\int T^{-1}\left(\frac{\partial \mathcal{M}}{\partial r_{+}}\right)_{Q_{i}} d r_{+}+S_{0},
\end{aligned}
$$

where the integration constant $S_{0}$ does not depend on $r_{+}$, but possibly depends on the coupling constants and the spacetime dimension. It should be noted that in the present approach it is necessary to retain in the line element all the terms proportional to $\varepsilon^{2}$ also. After some algebra one obtains the expression describing the entropy, which for $S_{0}=0$ coincides with the one given by Eq. (41).

The integration constant can be determined from the physical requirement that the entropy vanishes when the horizon radius shrinks to zero 32, 46]. For the Lovelock theory it has been 
shown that this condition leads to the results which are identical with those obtained within the framework of the Euclidean approach. For $d>4$ this procedure gives $S_{0}=0$.

The entropy as given by Eq. (411) is expressed in terms of the exact location of the event horizon, $r_{+}$, and therefore it depends on the full set of the coupling constants. However, according to our previous discussion, one can easily reduce their number by a suitable choice of representation. Indeed, expressing the entropy in terms of the total mass as seen by a distant observer (or, equivalently, $C$ ) reduces the number of remaining coupling constants to three. To demonstrate this, let us substitute $r_{+}$given by Eq.(28) into Eq.(41) and retain the terms up to second order in $\varepsilon$. After some rearrangement, one obtains

$$
\begin{aligned}
S= & \frac{\omega_{d}}{4 G_{D}}(2 C)^{d /(d-1)}+\frac{\varepsilon \omega_{d}}{4 a G_{D}}(2 C)^{(d-2) /(d-1)} d^{2} b_{3}+\frac{\varepsilon^{2} \omega_{d}}{8 a G_{D}}(2 C)^{(d-4) /(d-1)}\left[c_{7}\left(2+2 d-3 d^{2}+d^{3}\right)\right. \\
& \left.-\frac{1}{4} c_{8}\left(4+5 d-6 d^{2}+d^{3}\right)-\frac{1}{a} b_{3}^{2}(d-2)^{2}(1+2 d)\right] .
\end{aligned}
$$

The entropy described by the above equation depends only on $b_{3}, c_{7}$ and $c_{8}$ as expected.

Now, let us compare (44) with the analogous result constructed by Lu and Wise 20]. Putting in (44) $d=2$ and $G_{D}=G$, one has

$$
S=4 \pi G M^{2}+64 \pi^{2} \varepsilon b_{3}+\frac{2 \pi^{2}}{G^{2} M^{2}}\left(4 c_{4}+c_{8}\right) \varepsilon^{2} .
$$

Inspection of (45) shows that it contains the term proportional to $b_{3}$, which is absent in the Lu-Wise paper. This can be easily understood as Lu and Wise ignored the Gauss-Bonnet term, which, in four dimensions, does not affect black hole solution of the field equations. It affects, of course, the action itself and consequently the entropy, leading to appearance of a constant term in $S$ that is independent of $\mathcal{M}$.

Finally, let us restrict values of the coefficients to its Lovelock combinations. After some manipulations it could be shown, that (41) reduces to a simple expression

$$
S=\frac{r_{+}^{d} \omega_{d}}{4 G_{D} \alpha_{1}}\left[\alpha_{1}+\frac{2 \alpha_{2}}{r_{+}^{2}} d(d-1)+\frac{3 \alpha_{3}}{r_{+}^{4}} d(d-3)(d-2)(d-1)\right],
$$

which is identical with the exact result obtained from a general formula [32, 47]

$$
S=\frac{\omega_{d} r_{+}^{d}}{4 G_{D}} \sum_{n=1}^{m} \frac{n d}{d+2-2 n} \hat{c}_{n}\left(r_{+}^{-2}\right)^{n-1}
$$

for $m=3$. 


\section{FINAL REMARKS}

So far we have considered the boundary conditions of the second type only. Now, we shall briefly examine appropriate solution constructed with the aid of the conditions (17) and $\psi(\infty)=0$. Since both solutions are not independent one can treat the solution of the one type as the useful check of the other. Before we proceed further let us analyse some general features of the function $M(r)$. Nature of the problem and our previous analysis suggests that the solution has the form

$$
M(r)=\tilde{M}(r)+C_{1},
$$

with $\tilde{M}(\infty)=0$ and $\tilde{M}\left(r_{+}\right)=r^{d-1} / 2-C_{1}$, and, consequently, the total mass of the system as seen by a distant observer, $\mathcal{M}$, can be obtained from

$$
M(\infty)=C_{1}=\frac{8 \pi G_{D}}{d \omega_{d}} \mathcal{M}
$$

where $C_{1}$ is expressed, by construction, in terms of the exact location of the event horizon. One expects, therefore, that $C_{1}=C$, where $C$ is given by Eq. (38), and this equality can be treated as a consistency check. Repeating calculations order by order with the new boundary conditions, after some algebra it can be shown that the function $M(r)$ can be written as

$$
\begin{aligned}
M(r)= & -\frac{b_{3}}{2 a}(d-2)(d-1) \frac{r_{+}^{2 d-2}}{r^{d+1}}-\frac{b_{3}^{2}}{a^{2}}(d-2)^{2}(d-1)^{2} \frac{r_{+}^{2 d-4}}{r^{d+1}} \\
& +A_{1}(r) \frac{r_{+}^{2 d-2}}{r^{d+3}}+A_{2}(r) \frac{r_{+}^{3 d-3}}{r^{2 d+2}}+C_{1},
\end{aligned}
$$

where $C_{1}$ is given by Eq. (38),

$$
\begin{aligned}
A_{1}(r)= & \frac{(d-1)(d+1)^{2}}{a}\left[-2 d c_{3}+\frac{1}{4}(2-3 d) c_{6}-3(d-1) c_{7}+\frac{3}{4}(d-1) c_{8}\right] \\
& +\frac{(d-2)(d-1)(d+1)^{2}}{a^{2}}\left(3 b_{2} b_{3}+4 b_{1} b_{3}+8 b_{3}^{2}\right),
\end{aligned}
$$

and

$$
\begin{aligned}
A_{2}(r)= & \frac{(d-1)}{a}\left[\frac{c_{3}}{2} d(d+1)(3 d+5)-\frac{c_{6}}{4}(d+1)\left(3-3 d-2 d^{2}\right)\right. \\
& \left.-\frac{c_{7}}{4}\left(10+16 d-21 d^{2}-7 d^{3}\right)+\frac{c_{8}}{4}\left(2+25 d-24 d^{2}-7 d^{3}\right)\right] \\
& -\frac{1}{a^{2}}(d-2)(d-1)\left[2 b_{2} b_{3}(d+1)(d+2)+b_{1} b_{3}(d+1)(3 d+5)\right. \\
& \left.+b_{3}^{2}\left(9+19 d+4 d^{2}\right)\right] .
\end{aligned}
$$


Similar calculations carried out for the function $\psi(r)$ yield

$$
\begin{aligned}
\psi(r)= & \frac{2}{a^{2}}(d-2)(d-1)(d+1)\left[b_{2} b_{3}(d+2)+b_{1} b_{3}(2 d+3)+b_{3}^{2}(2 d+5)\right] \frac{r_{+}^{2 d-2}}{r^{2 d+2}} \\
& -\frac{(d-1)(d+1)}{a}\left[c_{3} d(3+2 d)-c_{6}\left(1-2 d-d^{2}\right)-\frac{3}{2} c_{7}\left(2-2 d-d^{2}\right)\right. \\
& \left.+\frac{3}{8} c_{8}\left(2-3 d-d^{2}\right)\right] \frac{r_{+}^{2 d-2}}{r^{2 d+2}} .
\end{aligned}
$$

Since, by assumption, the radius of the event horizon is treated as the exact quantity now, the thus derived line element may be easily employed in construction of the entropy. First, observe that the Hawking temperature calculated with the aid of the Eq. (30) is given in terms of $r_{+}$and depends on all relevant coefficients. On the other hand the relation (31) is independent of $b_{1}, b_{2}, c_{4}$ and $c_{5}$. This behaviour can be ascribed to the possibility to remove the dependence of the line element on this very coefficients at the expense of modifications of the equations of motion of test particles. Indeed, it could be demonstrated that by means of the field redefinition of the form

$$
g_{a b} \rightarrow g_{a b}+\varepsilon A_{a b}^{(1)}+\varepsilon^{2} A_{a b}^{(2)},
$$

where

$$
A_{a b}^{(1)}=q_{1}^{(1)} R g_{a b}+q_{2}^{(1)} R_{a b}
$$

and

$$
\begin{aligned}
A_{a b}^{(2)} & =q_{1}^{(2)} R^{2} g_{a b}+q_{2}^{(2)} R R_{a b}+q_{3}^{(2)} g_{a b} R_{c d e f} R^{c d e f}+q_{4}^{(2)} g_{a b} R_{c d} R^{c d} \\
& +q_{5}^{(2)} R_{a c} R_{b}^{c}+q_{6}^{(2)} R_{a c d e} R_{b}{ }^{c d e}
\end{aligned}
$$

one can remove all the terms in the action except these proportional to the parameters $b_{3}, c_{7}$ and $c_{8}$. The coefficients $q_{i}^{(1)}$ and $q_{k}^{(2)}$ can be determined by solving, at each order of the expansion, the appropriate systems of equations. As the result of the field redefinition (54), one obtains two additional terms $R \square R$ and $R_{a b} \square R^{a b}$, which can also be removed from the action functional. It should be noted, however, that such terms appear naturally in the effective action of the quantized massive fields in a large mass limit [48, 49, 50].

The geodesic equation after the field redefinition becomes

$$
\begin{aligned}
\frac{d^{2} x^{i}}{d s^{2}} & +\Gamma_{j k}^{i} \frac{d x^{j}}{d s} \frac{d x^{k}}{d s}+\varepsilon g^{i m}\left(A_{m k ; j}^{(1)}-\frac{1}{2} A_{j k ; m}^{(1)}\right) \frac{d x^{j}}{d s} \frac{d x^{k}}{d s} \\
& +\varepsilon^{2} g^{i m}\left(A_{m k ; j}^{(2)}-\frac{1}{2} A_{j k ; m}^{(2)}\right) \frac{d x^{j}}{d s} \frac{d x^{k}}{d s}-\varepsilon^{2} A^{(1) i m}\left(A_{m k ; j}^{(1)}-\frac{1}{2} A_{j k ; m}^{(1)}\right) \frac{d x^{j}}{d s} \frac{d x^{k}}{d s}=0,
\end{aligned}
$$


where $s$ is the affine parameter of the original metric. Now, one can repeat the arguments of Ref. 20]. Both $\mathcal{M}$ and $T$ can be measured at infinity and do not depend on the particular form of the equations of motion. Consequently, the temperature mass relation is independent of the removed terms. However, to determine the radius of the event horizon one performs local measurements and the equations of motion of test particles are important.

It could be easily seen that, as expected, the entropy is precisely the same for both choices of boundary conditions and is described by the formula (41). Since the calculations for both types of the boundary conditions have been carried out independently, this equality may be regarded as the additional consistency check.

\section{APPENDIX}

In this Appendix we list some formulas used in Section IV. The expansions of the components of the Riemann tensor and some of its contractions up to the first order in $\varepsilon$, which are necessary in calculation of the entropy, are given by

$$
\begin{gathered}
R_{t i}{ }^{t j}=R_{r i}{ }^{r j}=-\frac{(d-1)}{2 r_{+}^{2}} \delta_{i}^{j}+\varepsilon b_{3} \frac{(d-2)(d-1)(d+1)}{2 a r_{+}^{4}} \delta_{i}^{j}+O\left(\varepsilon^{2}\right), \\
R_{i j}{ }^{k l}=\frac{1}{r_{+}^{2}}\left(\delta_{i}^{k} \delta_{j}^{l}-\delta_{i}^{l} \delta_{j}^{k}\right)+O\left(\varepsilon^{2}\right) \\
R_{t r}{ }^{t r}=\frac{d(d-1)}{2 r_{+}^{2}}-\varepsilon b_{3} \frac{3(d-2)(d-1) d(d+1)}{2 a r_{+}^{4}}+O\left(\varepsilon^{2}\right) \\
R_{t}^{t}=R_{r}^{r}=-\varepsilon b_{3} \frac{(d-2)(d-1) d(d+1)}{2 a r_{+}^{4}}+O\left(\varepsilon^{2}\right)
\end{gathered}
$$

and

$$
R=-\varepsilon b_{3} \frac{(d-2)(d-1) d(d+1)}{2 a r_{+}^{4}}+O\left(\varepsilon^{2}\right)
$$

where $i, j, k, l=2, \ldots, d+1$.

[1] D. Lovelock, J. Math. Phys. 12, 498 (1971). 
[2] B. Zwiebach, Phys. Lett. B156, 315 (1985).

[3] B. Zumino, Phys. Rept. 137, 109 (1986).

[4] J. Z. Simon, Phys. Rev. D41, 3720 (1990).

[5] L. Parker and J. Z. Simon, Phys. Rev. D47, 1339 (1993), gr-qc/9211002.

[6] H. J. Bhabha, Phys. Rev. 70, 759 (1946).

[7] S. Deser and A. V. Ryzhov, Class. Quant. Grav. 22, 3315 (2005), gr-qc/0505039.

[8] S. A. Fulling, R. C. King, B. G. Wybourne, and C. J. Cummins, Class. Quant. Grav. 9, 1151 (1992).

[9] K. S. Stelle, General Relativity and Gravitation 9, 353 (1978).

[10] K. S. Stelle, Phys. Rev. D (3) 16, 953 (1977).

[11] N. H. Barth and S. M. Christensen, Phys. Rev. D 28, 1876 (1983).

[12] A. Economou and C. O. Lousto, Phys. Rev. D49, 5278 (1994), gr-qc/9310021.

[13] M. Campanelli, C. O. Lousto, and J. Audretsch, Phys. Rev. D49, 5188 (1994), gr-qc/9401013.

[14] M. Campanelli, C. O. Lousto, and J. Audretsch, Phys. Rev. D51, 6810 (1995), gr-qc/9412001.

[15] R. C. Myers (1998), gr-qc/9811042.

[16] J. Matyjasek and D. Tryniecki, Phys. Rev. D69, 124016 (2004), gr-qc/0402098.

[17] S. Nojiri and S. D. Odintsov, Phys. Lett. B521, 87 (2001), hep-th/0109122.

[18] R. C. Myers, Nucl. Phys. B289, 701 (1987).

[19] J. Callan, Curtis G., R. C. Myers, and M. J. Perry, Nucl. Phys. B311, 673 (1989).

[20] M. Lu and M. B. Wise, Phys. Rev. D47, 3095 (1993), gr-qc/9301021.

[21] A. Dobado and A. Lopez, Phys. Lett. B316, 250 (1993), hep-ph/9309221.

[22] S. Nojiri and S. D. Odintsov, Phys. Lett. B523, 165 (2001), hep-th/0110064.

[23] S. Nojiri and S. D. Odintsov, Phys. Rev. D66, 044012 (2002), hep-th/0204112.

[24] Y. M. Cho and I. P. Neupane, Phys. Rev. D66, 024044 (2002), hep-th/0202140.

[25] I. P. Neupane, Phys. Rev. D67, 061501 (2003), hep-th/0212092.

[26] M. H. Dehghani and M. Shamirzaie, Phys. Rev. D72, 124015 (2005), hep-th/0506227.

[27] F. R. Tangherlini, Nuovo Cimento 27, 636 (1963).

[28] R. C. Myers and M. J. Perry, Ann. Phys. 172, 304 (1986).

[29] D. G. Boulware and S. Deser, Phys. Rev. Lett. 55, 2656 (1985).

[30] J. T. Wheeler, Nucl. Phys. B268, 737 (1986).

[31] J. T. Wheeler, Nucl. Phys. B273, 732 (1986).

[32] R.-G. Cai, Phys. Lett. B582, 237 (2004), hep-th/0311240.

[33] J. Matyjasek, Phys. Rev. D61, 124019 (2000), gr-qc/9912020.

[34] J. Matyjasek, Phys. Rev. D63, 084004 (2001), gr-qc/0010097.

[35] J. Matyjasek and O. B. Zaslavsky, Phys. Rev. D64, 104018 (2001), gr-qc/0102109.

[36] S. Deser and B. Tekin, Class. Quant. Grav. 20, 4877 (2003), gr-qc/0306114.

[37] S. Deser, J. Franklin, and B. Tekin, Class. Quant. Grav. 21, 5295 (2004), gr-qc/0404120.

[38] S. Deser and J. Franklin, Am. J. Phys. 73, 261 (2005), gr-qc/0408067. 
[39] J. Preskill, P. Schwarz, A. D. Shapere, S. Trivedi, and F. Wilczek, Mod. Phys. Lett. A6, 2353 (1991).

[40] C. F. E. Holzhey and F. Wilczek, Nucl. Phys. B380, 447 (1992), hep-th/9202014.

[41] R. M. Wald, Phys. Rev. D48, 3427 (1993), gr-qc/9307038.

[42] V. Iyer and R. M. Wald, Phys. Rev. D50, 846 (1994), gr-qc/9403028.

[43] T. Jacobson, G. Kang, and R. C. Myers, Phys. Rev. D49, 6587 (1994), gr-qc/9312023.

[44] T. Jacobson, G. Kang, and R. C. Myers, Phys. Rev. D52, 3518 (1995), gr-qc/9503020.

[45] M. Visser, Phys. Rev. D48, 5697 (1993), hep-th/9307194.

[46] T. Clunan, S. F. Ross, and D. J. Smith, Class. Quant. Grav. 21, 3447 (2004), gr-qc/0402044.

[47] T. Jacobson and R. C. Myers, Phys. Rev. Lett. 70, 3684 (1993), hep-th/9305016.

[48] I. G. Avramidi, Nucl. Phys. B355, 712 (1991).

[49] I. G. Avramidi, Theor. Math. Phys. 79, 494 (1989).

[50] I. G. Avramidi (1986), hep-th/9510140. 